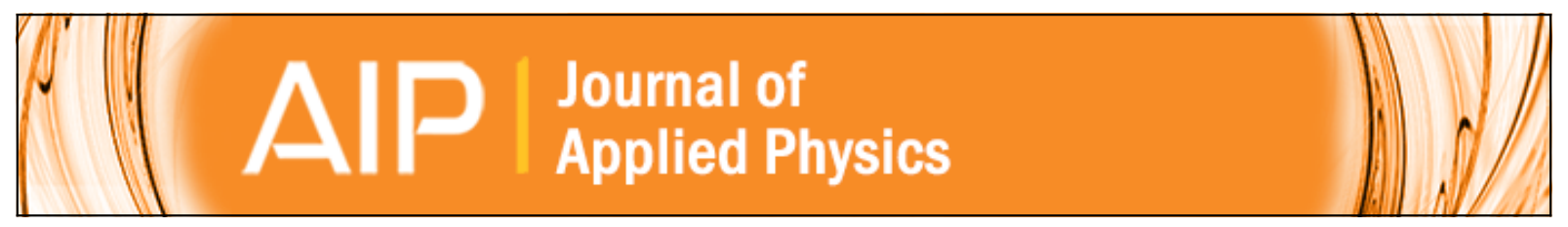

Energy exchange during stimulated Raman scattering of a relativistic laser in a plasma

D. N. Gupta, M. S. Hur, and H. Suk

Citation: Journal of Applied Physics 100, 103101 (2006); doi: 10.1063/1.2384808

View online: http://dx.doi.org/10.1063/1.2384808

View Table of Contents: http://scitation.aip.org/content/aip/journal/jap/100/10?ver=pdfcov

Published by the AIP Publishing

Articles you may be interested in

Third harmonic stimulated Raman backscattering of laser in a magnetized plasma

Phys. Plasmas 20, 092108 (2013); 10.1063/1.4821033

Relativistic Raman backscattering in an electron beam with ion-channel guiding and its application in freeelectron laser

Phys. Plasmas 12, 113106 (2005); 10.1063/1.2131048

Stimulated Raman scattering of relativistic laser beam in plasmas

Phys. Plasmas 12, 042302 (2005); 10.1063/1.1856480

Stimulated scattering and harmonic generation from relativistically intense laser light in plasma

Phys. Plasmas 10, 1135 (2003); 10.1063/1.1559732

Stimulated Brillouin and Raman scattering from a randomized laser beam in large inhomogeneous collisional plasmas. II. Model description and comparison with experiments

Phys. Plasmas 8, 1636 (2001); 10.1063/1.1357218

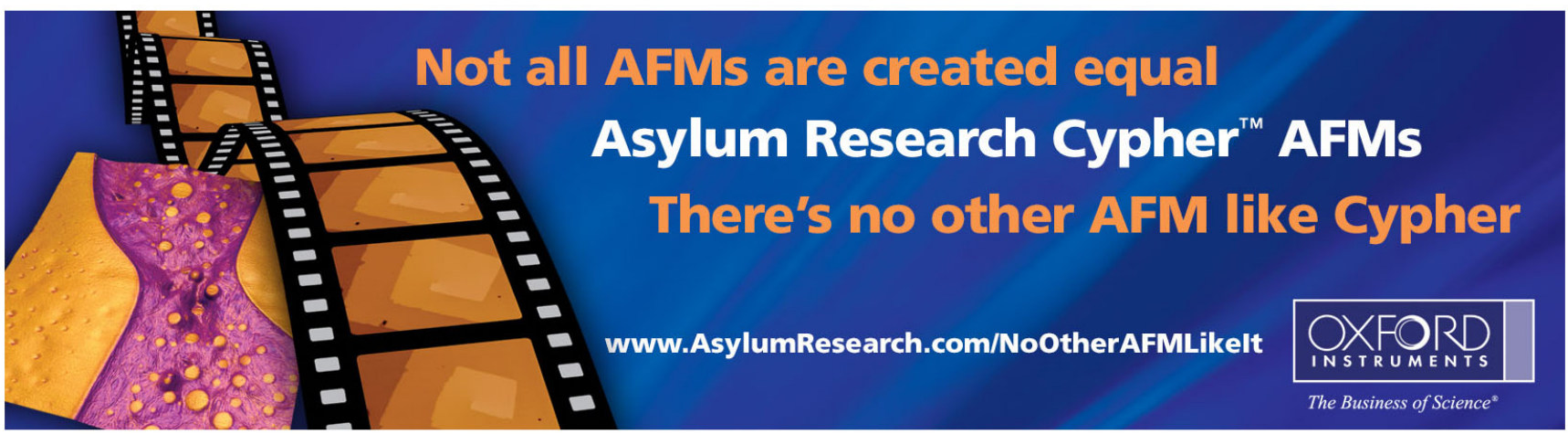




\title{
Energy exchange during stimulated Raman scattering of a relativistic laser in a plasma
}

\author{
D. N. Gupta, ${ }^{a}$ M. S. Hur, and H. Suk ${ }^{\text {b) }}$ \\ Center for Advanced Accelerators, Korea Electrotechnology Research Institute, Changwon 641-120, Korea
}

(Received 24 January 2006; accepted 2 September 2006; published online 20 November 2006)

\begin{abstract}
Energy exchange between pump and daughter waves during the stimulated Raman scattering process in a plasma is investigated, including the effect of a damping coefficient of electron-ion collision at different initial three-wave phases. To obey the energy and momentum conservations, the resonance conditions are satisfied at an optimal initial phase difference between the interacting waves. The amplitudes of the interacting waves exhibit behaviors such as a parametric oscillator. The variations in initial three-wave phase difference generate a phase mismatch, which enhances the rate of the amplitude variations of the interacting waves. The relativistic mass effect modifies the dispersion relations of the interacting waves, and consequently the energy exchange during the stimulated Raman scattering is affected. The collisional damping in the plasma is shown to have an important effect on the evolution of the interacting waves. (C) 2006 American Institute of Physics.
\end{abstract} [DOI: $10.1063 / 1.2384808$ ]

\section{INTRODUCTION}

Stimulated Raman scattering (SRS) of high power electromagnetic waves in plasmas has renewed the research interest because of its pivotal role in generation of large amplitude plasma waves and subsequent electron acceleration. ${ }^{1-5}$ It is relevant to laser driven fusion, ${ }^{6}$ laser acceleration of electrons and ions, ${ }^{7,8}$ ion Coulomb explosion, ${ }^{9}$ and x-ray lasers. ${ }^{10}$ In SRS, the electromagnetic pump wave excites a Langmuir wave and an electromagnetic sideband wave. The density perturbation associated with the Langmuir wave couples with the electron quiver motion by the pump wave to produce a nonlinear current driving of the sideband. Then the pump and sideband waves exert a ponderomotive force on electrons to drive the Langmuir wave even more strongly. ${ }^{11}$ SRS could be detrimental in inertial confinement fusion experiments because the scattered light decreases the laser-plasma coupling and may affect the irradiation symmetry. In addition, the Langmuir waves accelerate the electrons which may preheat the fusion fuel.

In a low-density plasma Raman forward scattering is a more relevant process. ${ }^{12-14}$ It gives rise to a high amplitude, high phase velocity plasma wave that can accelerate the electrons to a very high energy. The forward Raman instability, in the case of a laser with a finite spot size, couples to the self-focusing and produces a plasma wave of much higher amplitude. ${ }^{15,16}$ Mori and Decker ${ }^{17}$ have analyzed the spatialtemporal growth of the Raman scattering from a set of the equations valid for weakly relativistic pumping. They presented an exact solution for the evolution of Raman scattering with quasistatic approximation. The recent development of ultrashort and ultraintense laser pulse technology such as the chirped-pulse amplification ${ }^{18}$ has led to the generation of laser intensities at higher orders of magnitude. In this regime, electrons oscillate at relativistic velocities in laser fields that

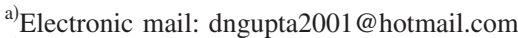

${ }^{\mathrm{b})}$ Electronic mail: hysuk@keri.re.kr
}

exceed $10^{11} \mathrm{~V} / \mathrm{cm}$, which results in a relativistic mass increase. Stimulated Raman scattering driven by the relativistic intense laser pulses has been studied by many authors. ${ }^{19-21}$ Modena ${ }^{22}$ has observed experimentally the Raman forward scattering in the relativistic regime and presented various results.

We study the stimulated Raman forward scattering of a relativistic laser by using a linear perturbation theory. The theory includes the effect of damping coefficient of electronion collision and the effect of three-wave phase difference. The dispersion relations of the pump and daughter waves will be modified due to the relativistic mass change and the damping coefficient. They also depend on the amplitudes of the incident laser and the scattered light. The modified dispersion relations affect the temporal evolution of the stimulated Raman scattering of a laser. The initial three-wave phases also change the temporal development of SRS. This paper is organized as follows. In Sec. II, we establish the dispersion relations of the SRS waves which include the effect of the electron damping coefficient and relativistic mass change. The coupled mode equations for the laser pump wave, the scattered electromagnetic wave, and the plasma wave in a plasma are derived, and the numerical results are discussed. The conclusions are presented in the last section.

\section{TEMPORAL EVOLUTION OF STIMULATED RAMAN SCATTERING}

Let us consider a high intensity laser propagating in a plasma with a density of $n_{0}$ and an electron temperature of $T_{e}$, where the laser has an electric field,

$$
\mathbf{E}_{0}=\hat{x} A_{0}(t) \exp \left[-i\left(\omega_{0} t-k_{0} x\right)\right] .
$$

In the stimulated Raman scattering process, an electromagnetic pump wave $\left(\omega_{0}, \mathbf{k}_{0}\right)$ couples with an electrostatic perturbation $\quad \mathbf{E}=-\boldsymbol{\nabla} \phi=\hat{z} A(t) \exp [-i(\omega t-k x)] \quad[$ an electron plasma wave $(\omega, \mathbf{k})]$ and generates a scattered sideband $\mathbf{E}_{1}$ $=\hat{x} A_{1}(t) \exp \left[-i\left(\omega_{1} t \pm k_{1} x\right)\right]$ an electromagnetic wave $\left(\omega_{1}, \mathbf{k}_{1}\right)$; 
$\left.\left(\omega_{1}=\omega_{0}-\omega, \mathbf{k}_{1}=\mathbf{k}_{0} \mp \mathbf{k}\right)\right]$. The upper sideband $\left(\omega+\omega_{0}\right)$ is neglected as it is considered to be off resonance for the nonlinear interaction under investigation. Here the symbol $\mp$ corresponds to forward- and backscattering electromagnetic waves. We consider the Raman forward scattering and establish the dispersion relation without the collisional damping effect.

From the linear perturbation theory, the oscillatory velocities of electrons imparted by the interacting waves are $\boldsymbol{v}_{0}=e \mathbf{E}_{0} / m i \gamma_{0} \omega_{0}, \boldsymbol{v}_{1}=e \mathbf{E}_{1} / m i \gamma_{1} \omega_{1}$, and $\boldsymbol{v}=e \mathbf{E} / m i \gamma \omega$, where $\gamma_{0}=\left(1+a_{0}^{2} / 2\right)^{1 / 2}, \quad \gamma_{1}=\left(1+a_{1}^{2} / 2\right)^{1 / 2}$, and $\gamma=\left(1+a^{2} / 2\right)^{1 / 2}, a_{0}$ $=e A_{0} / m \omega_{0} c, a_{1}=e A_{1} / m \omega_{1} c$, and $a=e A / m \omega c,-e$ and $m$ are the electron's charge and mass, respectively, and $c$ is the velocity of light in vacuum. When the amplitudes of interacting waves are comparable to each other, the effective relativistic factor ${ }^{23}$ may be taken as $\gamma_{0}^{0}=\left(1+a_{0}^{2} / 2+a_{1}^{2} / 2\right.$ $\left.+a^{2} / 2\right)^{1 / 2}$.

The pump and sideband waves exert a ponderomotive force on the electrons at $(\omega, \mathbf{k}), \mathbf{F}_{p}=e \boldsymbol{\nabla} \phi_{p}$, where $\phi_{p}$ $=-(m / 2 e) \gamma_{0}^{0} \boldsymbol{v}_{0} \boldsymbol{v}_{1}$. The electron density perturbation at $(\omega, \mathbf{k})$ due to $\phi$ and $\phi_{p}$ is

$$
n=\frac{k^{2}}{4 \pi e} \chi_{e}\left(\phi+\phi_{p}\right)
$$

where $\chi_{e}=-\left(\omega_{p}^{2}+3 k^{2} v_{\mathrm{th}}^{2}\right) / \omega^{2} \gamma_{0}^{0}$ is the electron susceptibility, $\omega_{p}=\left(4 \pi n_{0} e^{2} / m\right)^{1 / 2}$ is the plasma frequency, and $v_{\text {th }}$ $=\left(2 T_{e} / m\right)^{1 / 2}$ is the electron thermal velocity. Using this expression in the Poisson's equation $\nabla^{2} \phi=4 \pi n e$, we obtain

$$
\varepsilon \phi=-\chi_{e} \phi_{p},
$$

where $\varepsilon=1+\chi_{e}$. In the absence of the pump $\varepsilon=0$, the dispersion relation of the relativistic plasma wave can be written as $\omega^{2}=\left(\omega_{p}^{2}+3 k^{2} v_{t h}^{2}\right) / \gamma_{0}^{0}$.

The electron density perturbation at $(\omega, \mathbf{k})$ couples with oscillatory velocity due to the pump to produce a nonlinear current at $\left(\omega_{1}, \mathbf{k}_{1}\right)$,

$$
\mathbf{J}_{1}^{\mathrm{Nl}}=-\frac{1}{2} n e \boldsymbol{v}_{0}^{*}=\frac{e}{2} v_{0}^{*} \frac{k^{2} \phi}{4 \pi e} .
$$

Following the standard method ${ }^{24}$ the dispersion relation of sideband electromagnetic wave can be written as

$$
D_{1} \mathbf{E}_{1}=-\frac{i \omega_{1}}{2} k^{2} \phi \boldsymbol{v}_{0}^{*},
$$

where $D_{1}=\omega_{1}^{2}-\omega_{p}^{2} / \gamma_{0}^{0}-k_{1}^{2} c^{2}$.

To include the effect of damping, we add the term $-\nu \partial \mathbf{E}_{1} / \partial t$ in the left hand side of Eq. (5), where $\nu=\nu_{\mathrm{ei}}$ is the energy damping rate for the sideband wave in the collisional plasma, and $\nu_{\mathrm{ei}}$ is the electron-ion collision frequency. In the presence of the damping coefficient $(\nu)$ due to electron-ion collisions, $D_{1}$ can be expressed as

$$
D_{1}=\omega_{1}^{2}-\omega_{p}^{2} / \gamma_{0}^{0}-k_{1}^{2} c^{2}+i \nu_{\mathrm{ei}} \omega_{1} \text {. }
$$

The damping coefficient of electron-ion collision changes the dispersion relations of the SRS waves, which further affects the temporal evolution of the SRS waves. In the absence of pump $\left(D_{1}=0\right)$, the dispersion relation of the relativistic electromagnetic sideband wave can be obtained as $\omega_{1}^{2}=\omega_{p}^{2} / \gamma_{0}^{0}+k_{1}^{2} c^{2}-i \nu_{\mathrm{ei}} \omega_{1}$. Using Eqs. (3) and (5), we obtain the nonlinear dispersion relation of SRS,

$$
\varepsilon D_{1}=\frac{k^{2}\left|v_{0}\right|^{2}}{4 \gamma_{0}^{0}} \frac{\omega_{p}^{2}}{\omega^{2}} \text {. }
$$

By using the above equations, the standard expression of the maximum growth rate of the SRS can be written as ${ }^{25}$ $\Gamma_{m}=\left(\omega_{p}^{2} / \omega_{0}\right) a_{0} / 2 \sqrt{2}-\nu$. Here the pump amplitude $a_{0}$ is time dependent; hence, the growth rate varies according to the pump amplitude.

Replacing $\omega_{1}$ by $\omega_{1}+i \partial / \partial t$ and by using the Taylor expansion of $D_{1}\left(\omega_{1}, k_{1}\right)$ around $t=0$, one can express $D_{1}\left(\omega_{1}, k_{1}\right)=-i\left[\partial D_{1}\left(\omega_{1}, k_{1}\right) / \partial \omega_{1}\right](\partial / \partial t)$, where $D_{1}\left(\omega_{1}, k_{1}\right)_{t=0}$ $\ll \partial D_{1}\left(\omega_{1}, k_{1}\right) / \partial \omega_{1}$, and Eq. (7) can be written as ${ }^{11,26}$

$$
\frac{\partial A_{1}}{\partial t}=i \alpha_{1} A A_{0}^{*}-\frac{\nu_{\mathrm{ei}}}{2} A_{1} \text {. }
$$

In the same way, the behavior of the pump wave can be represented by the following equation:

$$
\frac{\partial A_{0}}{\partial t}=i \alpha_{0} A A_{1}^{*}-\frac{\nu_{\mathrm{ei}}}{2} A_{0} .
$$

Similarly, Eq. (3) gives

$$
\frac{\partial A}{\partial t}=i \alpha\left(\frac{\omega^{2}}{\omega_{p}^{2}}\right) \frac{\omega}{\omega_{1}} A_{0} A_{1}-\left(\frac{3 k^{2} v_{\mathrm{th}}^{2} \omega}{2 \omega_{p}^{2}}\right) A,
$$

where $\quad \alpha_{0}=e k_{0} / 4 m \gamma_{0}^{0} \omega_{0}, \quad \alpha_{1}=e k_{1} / 4 m \gamma_{0}^{0} \omega_{1}, \quad$ and $\quad \alpha$ $=-e k \chi_{e} / 2 m \omega_{0}$.

Expressing $A=(m \omega c / e) a(t) \exp [i \theta(t)], \quad A_{1}=\left(m \omega_{1} c / e\right)$ $\times a_{1}(t) \exp \left[i \theta_{1}(t)\right]$, and $A_{0}=\left(m \omega_{0} c / e\right) a_{0}(t) \exp \left[i \theta_{0}(t)\right]$ in the above equations and separating the real and imaginary parts, one obtains the following equation from Eqs. (9) and (10):

$$
\begin{aligned}
& \frac{\partial a_{0}}{\partial \tau}=-\frac{\alpha_{0}}{\alpha_{1}}\left(\frac{\omega}{\omega_{0}}\right) a a_{1} \sin \psi-\frac{\nu_{\mathrm{ei}}}{2 \omega_{0}}\left(\frac{\omega_{0} \gamma_{0}^{0}}{k c}\right) a_{0}, \\
& \frac{\partial a_{1}}{\partial \tau}=\frac{\alpha_{1}}{\alpha_{0}}\left(\frac{\omega}{\omega_{1}}\right) a a_{0} \sin \psi-\frac{\nu_{\mathrm{ei}}}{2 \omega_{0}}\left(\frac{\omega_{0} \gamma_{0}^{0}}{k c}\right) a_{1}, \\
& \frac{\partial a}{\partial \tau}=\frac{\alpha}{\alpha_{0}}\left(\frac{\omega_{1}}{\omega}\right) a_{0} a_{1} \sin \psi-6\left(\frac{k^{2} v_{\mathrm{th}}^{2}}{\omega_{p}^{2}}\right)\left(\frac{\omega \gamma_{0}^{0}}{k c}\right) a,
\end{aligned}
$$

and

$$
\begin{aligned}
\frac{\partial \psi}{\partial \tau}= & {\left[\frac{\alpha}{\alpha_{0}}\left(\frac{\omega_{1}}{\omega_{0}}\right) \frac{a_{0} a_{1}}{a}-\frac{\alpha_{0}}{\alpha_{1}}\left(\frac{\omega}{\omega_{0}}\right) \frac{a a_{1}}{a_{0}}\right.} \\
& \left.+\frac{\alpha_{1}}{\alpha_{0}}\left(\frac{\omega}{\omega_{1}}\right) \frac{a a_{0}}{a_{1}}\right] \cos \psi,
\end{aligned}
$$

where $\left(m \omega_{0} c \alpha_{0} / e\right) t=\tau, \psi=\theta-\theta_{0}-\theta_{1}$, and $\theta, \theta_{0}$, and $\theta_{1}$ are the phases of the plasma, pump, and sideband waves, respectively.

Equations (11)-(14) are coupled ordinary differential equations. These have been solved numerically by using the MATHEMATICA. All the numerical parameters are chosen for a Ti:sapphire-Nd:glass laser $\left(I \approx 5.7 \times 10^{17} \mathrm{~W} \mathrm{~cm}^{-2}\right)$ and the electron density $n_{0}=1.1 \times 10^{19} \mathrm{~cm}^{-3}\left(\omega_{p}=2 \times 10^{14} \mathrm{~s}^{-1}\right)$. We choose the laser frequency $\omega_{0}=2.4 \times 10^{15} \mathrm{~s}^{-1}$, sideband wave 

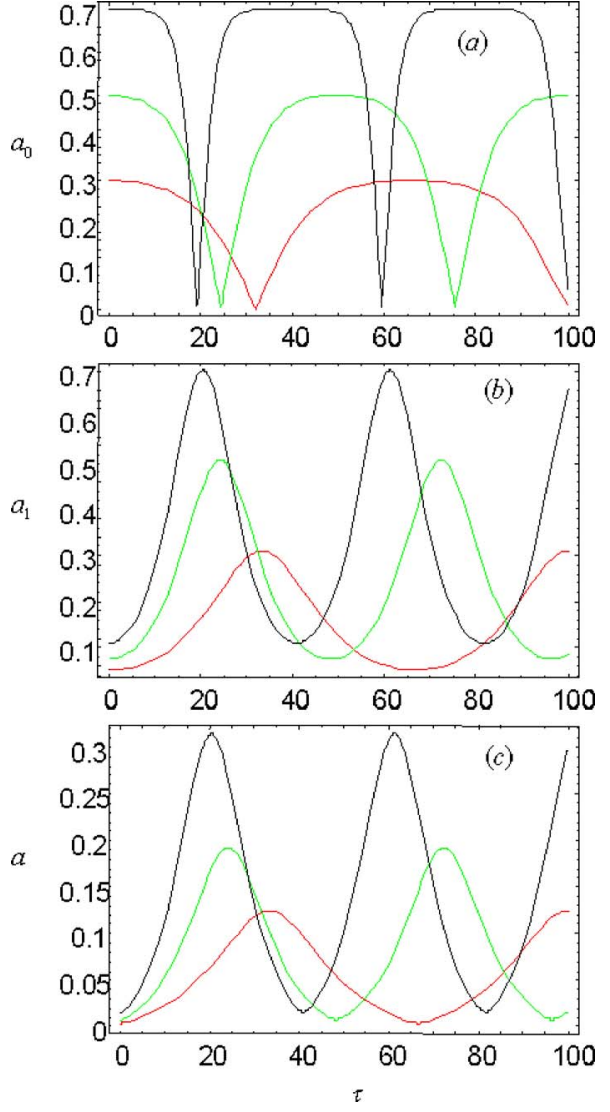

FIG. 1. (Color online) (a) Temporal evolution of the normalized pump wave amplitude $\left(a_{0}\right)$ (b) the normalized sideband wave amplitude $\left(a_{1}\right)$ and (c) the normalized plasma wave amplitude $(a)$ in the absence of damping losses. The black, green, and red lines are for $a_{0}(\tau=0)=0.7,0.5$, and 0.3 , respectively.

frequency $\omega_{1}=2.2 \times 10^{15} \mathrm{~s}^{-1}$, and $\nu_{\mathrm{ei}} / \omega_{0}=0.05,0.1$ and $k v_{\mathrm{th}} / \omega_{p}=0.07,0.09$ (corresponding to the electron temperatures of 20 and $50 \mathrm{eV}$, respectively). The initial conditions are $a_{0}=0.3,0.5,0.7, a_{1}=0.05, a=0.01$, and $\psi=90^{\circ}$ at initial normalized time $\tau=0$. These experimental parameters are available at Korea Electrotechnology Research Institute and feasible for laser-plasma interaction research nowadays. Here we have chosen the nonzero values of the initial amplitudes of the daughter waves for simplicity of mathematical calculations. Other initial amplitudes of the daughter waves will not make any changes in the behavior of the interacting waves.

First, we show the variations of time-dependent amplitudes of the pump wave $a_{0}$ [Fig. 1(a)], the sideband wave $a_{1}$ [Fig. 1(b)], and the plasma wave $a$ [Fig. 1(c)] in the absence of damping coefficient of electron-ion collision for different initial laser intensities $a_{0}=0.3$ (red line), $a_{0}=0.5$ (green line), and $a_{0}=0.7$ (black line). Here we choose a simplified case when initial three-wave phase is locked at $\psi=90^{\circ}$. The pump wave is responsible for SRS and diverts its energy to the daughter waves. In the result, the amplitude of the pump wave decreases with time in the beginning. As time goes, the amplitude of the pump wave increases after attaining a minimum. Oppositely, the amplitudes of the sideband wave and the plasma wave increase due to the seeding effect of the instability. The amplitudes of the sideband wave and the
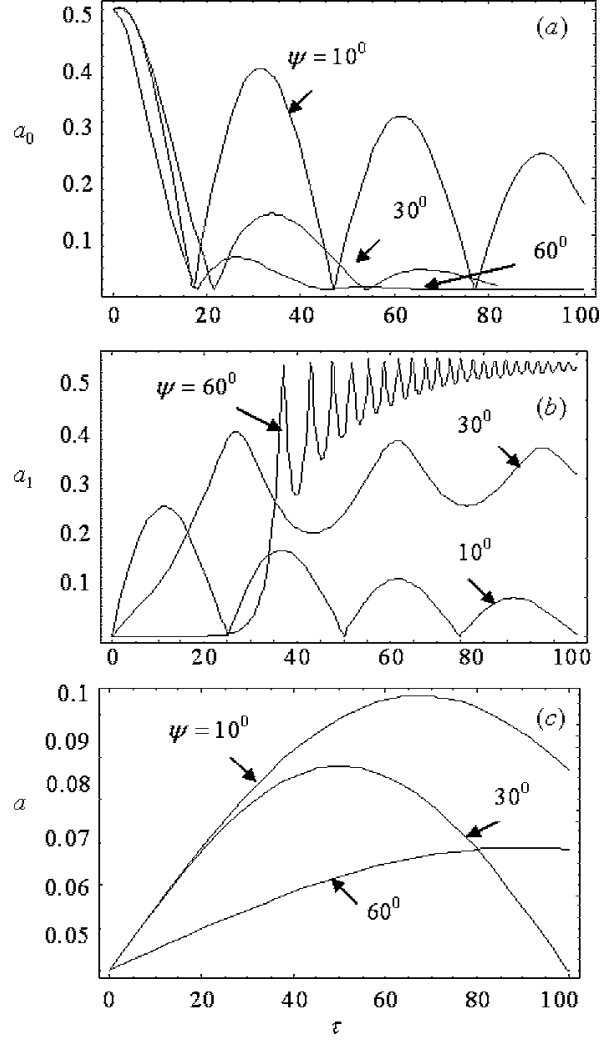

FIG. 2. (a) Temporal evolution of the normalized pump wave amplitude, (b) the normalized sideband wave amplitude, and (c) the normalized plasma wave amplitude in the presence of damping coefficient $\left(\nu_{\mathrm{ei}} / \omega_{0}=0.05\right)$ for $T_{e}=20 \mathrm{eV}$.

plasma wave decrease after attaining a peak. It is observed that the peaks of the amplitudes of decay waves occur when the pump wave amplitude gets minima and vice versa. The physics behind the oscillatory growths of the interacting waves can be understood as follows. As we know in the SRS process, the laser incident upon a plasma scatters into an electromagnetic sideband and a plasma wave. In this process, a part of the incident energy is scattered and a part is deposited into the electron plasma wave. This is a feedback process where the energy and the momentum are conserved. When initial three-wave phase is locked, there is no energy loss during SRS due to the resonant interactions. If the pump wave amplitude decreases, the daughter wave amplitude will increase because of the energy conservation. This process seems to be an analogy of the parametric oscillator.

At high laser powers, the plasma wave amplitude can be comparable to the sideband wave amplitude because of the significant growth of SRS. It is observed that the amplitude evolutions of the pump and daughter waves are affected sufficiently by the relativistic effect. The amplitude of the pump wave has the first minimum at time $\tau=30$ for initial laser intensity $a_{0}=0.3$. For a higher laser intensity $a_{0}=0.5$, it has the first minimum at earlier time $\tau=20$. Hence, the rate of amplitude variation increases due to the relativistic effect.

In Fig. 2, we see the effect of the three-wave phase on the temporal evolutions of the SRS waves in the presence of the damping coefficient of the electron-ion collision. Figure 2(a) shows the variation of the pump amplitude with time in the presence of the damping coefficient $\left(\nu_{\mathrm{ei}} / \omega_{0}=0.05\right)$ for 
different three-wave phases $\left(\psi=10^{\circ}, 30^{\circ}, 60^{\circ}\right)$. The pump amplitude decreases and finally tends to get a minimum value. For a small value of the three-wave phase difference $\left(\psi=10^{\circ}\right)$, the amplitude of the pump wave decreases to the first minimum, and then it shows an oscillatory behavior. For higher values of the three-wave phase differences $(\psi$ $=30^{\circ}, 60^{\circ}$ ), initially the amplitude of the pump wave shows the same behavior, but as time goes it decreases quickly to zero. The lost energy of the pump wave amplifies the SRS, which enhances the amplitudes of the sideband [Fig. 2(b)] and plasma waves [Fig. 2(c)]. The changes in three-wave phase differences also affect the temporal evolution of the daughter wave. For larger three-wave phase difference, the amplitude of the sideband wave increases continuously and reaches a saturation level. The reason for the saturation level attained by the sideband is the resonance mismatch introduced by the initial phase differences of the interacting waves. The damping of the plasma wave and the detuning of the resonance both act to stabilize the sideband wave against the loss of pump wave energy. Only a small fraction of the pump energy is taken by the plasma wave because of the resonance mismatch at a larger three-wave phase difference. Furthermore, the sideband retains an amount of energy for a larger three-wave phase difference. But for a smaller threewave phase difference, it cannot retain the energy longer. This means that the larger three-wave phase difference is useful to obtain a large amplitude of the SRS. On the other hand, the amplitude of the plasma wave initially increases due to the pumping by the pump wave and begins to lose its energy after attaining a peak. The effect of three-wave phase difference on the plasma wave amplitude evolution can be seen in Fig. 2(c). Following the energy conservation, it can be predicted that the plasma wave obtains a higher amplitude for a smaller three-wave phase difference.

Figure 3 shows the effect of the damping coefficient of electron-ion collision on the development of the amplitudes of the SRS waves at a certain three-wave phase difference $\left(\psi=10^{\circ}\right)$. The pump wave amplitude decreases rapidly for large damping coefficient. Also, the pump wave loses its energy in short time for the larger damping rate [Fig. 3(a)]. Figure 3(b) shows the same for the amplitude of sideband wave. The higher value of the damping coefficient shifts the peaks of the amplitude slightly, and the damping rate reduces the SRS amplification. In Fig. 3(c), we represent the temporal evolution of the plasma wave for the damping coefficient $\nu_{\mathrm{ei}} / \omega_{0}=0.05$ and the different electron thermal velocities $k v_{\text {th }} / \omega_{p}=0.07,0.09$. The larger electron thermal speed enhances the saturation level of the plasma wave amplitude.

\section{CONCLUSIONS}

We explored some physics to examine the relativistic effect, the effect of damping coefficient of the electron-ion collision, and the effect of three-wave phase change on temporal evolution of the stimulated Raman scattering of a laser in a plasma. Some numerical calculations were carried out to justify the results by choosing a simplified case when the phases of the SRS waves are locked. The amplitudes of the decay waves grow initially and acquire the peaks later. Dur-
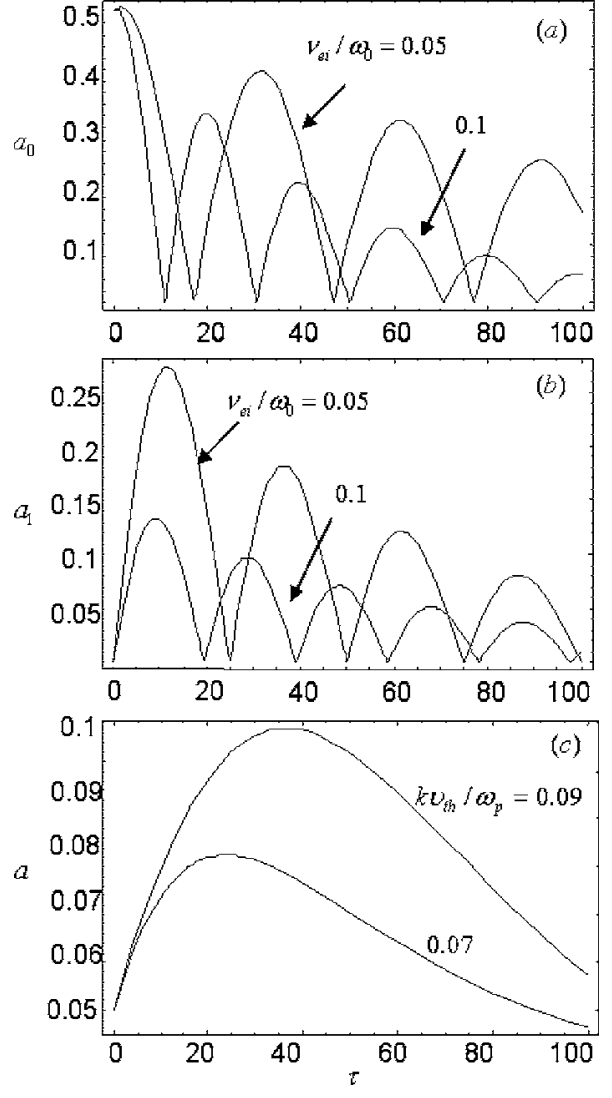

FIG. 3. (a) Temporal evolution of the normalized pump wave amplitude, (b) the normalized sideband wave amplitude, and (c) the normalized plasma wave amplitude in the presence of different damping coefficients $\left(\nu_{\mathrm{ei}} / \omega_{0}\right.$ $=0.05,0.1)$ for different electron thermal velocities $\left(k v_{\mathrm{th}} / \omega_{p}=0.07,0.09\right)$.

ing this period, the pump wave amplitude decreases and acquires a minimum. Beyond this time the amplitudes of the decay waves decrease, while the pump wave amplitude rises, giving rise to the exchanges of energies among the interacting waves. The relativistic mass effect plays a significant role in energy exchange between the pump and daughter waves in the SRS process. The unlocking of the initial three-wave phase difference changes the temporal evolution of the SRS waves. The resonance mismatching and the damping due to the collisions and thermal effects generate some losses during this process. Therefore, the interacting waves represent some different temporal behaviors in this case. According to the results, the amplitudes of the SRS waves strongly depend on the initial phases of three waves. The plasma collision damping changes the dispersion relations of the SRS waves and becomes an important factor for the development of the amplitudes of the SRS waves. The increasing three-wave phase difference and electron thermal velocity amplify the SRS; however, the increasing damping coefficient shows the reverse effect.

This theory is not applicable for Raman backscattering (RBS) because as the pump amplitude decreases and the daughter wave amplitude increases, the role of the pump and daughter waves is interchanged when the daughter wave amplitude overtakes the pump wave amplitude. In order for the energy to flow back into the pump wave by the large amplitude daughter wave, one needs upshifted stimulated Raman 
backscattering because the daughter wave is of lower frequency. Since this is a nonresonant process, it will not occur physically and there is no periodic energy exchange between the pump and daughter waves in this scheme. Hence, only the Raman forward scattering (RFS) may allow such an exchange.

\section{ACKNOWLEDGMENT}

This work was supported by the Korean Ministry of Science and Technology through the Creative Research Initiative Program/KOSEF.

${ }^{1}$ C. D. Decker, W. B. Mori, and T. Katsouleas, Phys. Rev. E 50, R3338 (1994).

${ }^{2}$ A. Modena et al., IEEE Trans. Plasma Sci. 24, 289 (1996).

${ }^{3}$ P. Sprangle, E. Esarey, A. Ting, and G. Joyce, Appl. Phys. Lett. 53, 2146 (1998).

${ }^{4}$ M. S. Hur, R. R. Lindberg, A. E. Charman, J. S. Wurtele, and H. Suk, Phys. Rev. Lett. 95, 115003 (2005).

${ }^{5}$ N. Hafz, M. S. Hur, G. H. Kim, C. Kim, I. S. Ko, and H. Suk, Phys. Rev. E 73, 016405 (2006).

${ }^{6}$ M. Tabak et al., Phys. Plasmas 1, 1626 (1994).

${ }^{7}$ G. Malka and J. L. Miquel, Phys. Rev. Lett. 77, 75 (1996); D. N. Gupta, H. Suk, and C. M. Ryu, Phys. Plasmas 12, 093110 (2005).

${ }^{8}$ H. J. Lee, K. H. Pae, H. Suk, and S. J. Hahn, Phys. Plasmas 11, 1726
(2004); J. Badziak, S. Glowacz, S. Jablonski, P. Parys, J. Wolowski, and H. Hora, Appl. Phys. Lett. 85, 3041 (2004).

${ }^{9}$ C. S. Liu and V. K. Tripathi, Phys. Plasmas 10, 4085 (2003), and references therein.

${ }^{10}$ P. Amendt, D. C. Eder, and S. C. Wilks, Phys. Rev. Lett. 66, 2589 (1991).

${ }^{11}$ C. S. Liu and V. K. Tripathi, Interaction of Electromagnetic Waves and Electron Beams with Plasmas (World Scientific, Singapore, 1994).

${ }^{12}$ C. S. Liu and V. K. Tripathi, Phys. Plasmas 3, 3410 (1996).

${ }^{13}$ G. Shvets and X. Li, Phys. Plasmas 8, 8 (2001).

${ }^{14}$ D. F. Gordon, H. Hafizi, R. F. Hubbard, and P. Sprangle, Phys. Plasmas 9, 1157 (2002).

${ }^{15}$ T. M. Antonsen, Jr., and P. Mora, Phys. Rev. Lett. 69, 2204 (1992).

${ }^{16}$ J. R. Penano, B. Hafizi, P. Sprangle, R. F. Hubbard, and A. Ting, Phys. Rev. E 66, 036402 (2002).

${ }^{17}$ W. B. Mori and C. D. Decker, Phys. Rev. Lett. 72, 1482 (1994).

${ }^{18}$ D. Strickland and G. Mourou, Opt. Commun. 55, 447 (1985).

${ }^{19}$ H. C. Barr, P. Mason, and D. M. Parr, Phys. Rev. Lett. 83, 1606 (1999).

${ }^{20}$ T. Yu-Hui, H. Shen-Sheng, Z. Chang-Xue, W. Yan-Qing, C. Jing, Z. FangChuan, Z. Yu-Zhe, and Xu Zhi-Zhan, Chin. Phys. 11, 50 (2002).

${ }^{21}$ C. B. Schroeder, E. Esarey, B. A. Shadwich, and W. P. Leemans, Phys. Plasmas 10, 285 (2003).

${ }^{22}$ A. Modena, IEEE Trans. Plasma Sci. 24, 289 (1996)

${ }^{23}$ B. L. Qian, Phys. Plasmas 7, 537 (2000).

${ }^{24}$ W. L. Kruer, The Physics of Laser Plasma Interactions (Addison-Wesley, New York, 1988).

${ }^{25}$ C. J. McKinstrie and R. Bingham, Phys. Fluids B 4, 2626 (1992).

${ }^{26} \mathrm{~K}$. Nishikawa and C. S. Liu, in Advances in Plasma Physics, edited by A. Simon and W. Thompson (Wiley, New York, 1976), Vol. 16 